\title{
Angular resolution of LISA
}

\author{
M Peterseim $\dagger$, O Jennrich $\dagger$, K Danzmann††, B F Schutz§ \\ $\dagger$ Institut für Atom- und Molekülphysik, Universität Hannover, Appelsstraße 2, 30167 Hannover, \\ Germany \\ $\ddagger$ MPI für Quantenoptik, Hans-Kopfermann-Straße 1, 85748 Garching, Germany \\ $\S$ University of Wales, College of Cardiff, UK \\ and \\ Albert-Einstein-Institut, MPI für Gravitationsphysik, Potsdam, Germany
}

Received 15 August 1996, in final form 14 February 1997

\begin{abstract}
LISA is a space-borne, laser-interferometric gravitational wave detector currently under study by the European Space Agency. We give a brief introduction about the main features of the detector, concentrating on its one-year orbital motion around the Sun. We show that the amplitude as well as the phase of a gravitational wave is modulated due to that motion, allowing us to extract information from the signal. The most common way to estimate the parameters which characterize a signal present in a noisy data stream is to use the matched filtering technique. A brief review of the theory of parameter estimation, based on the work of Finn and Cutler, will be given. We carried out a simulation of the detection of a monochromatic gravitational wave based on that theory and focusing on estimating the angular parameters of the source. The results of the semi-analytic calculations are presented in detail and interpreted to determine the angular resolution of LISA.
\end{abstract}

PACS numbers: $9780 \mathrm{G}, 9585 \mathrm{~S}, 0480 \mathrm{~N}, 9555 \mathrm{Y}$

\section{Introduction}

The LISA mission consists of six spacecraft forming a laser interferometer in a plane inclined $60^{\circ}$ with respect to the ecliptic and along a circle with a radius of $3 \times 10^{9} \mathrm{~m}$ [5]. Two spacecraft each will be placed at three points on this circle forming an equilateral triangle with baselines of $5 \times 10^{9} \mathrm{~m}$ which rotates clockwise around its centre, as viewed from the Sun, with a period of one year. The complete constellation describes an approximately circular orbit at a distance of $R=1 \mathrm{AU}$ from the Sun and trailing the Earth in its orbit by $20^{\circ}$. As a single, non-moving detector reveals no information about the directional parameters of the source of the gravitational wave, all the information about the source parameters is contained in the variation of the detector response that results from the described motion.

Firstly, the detected amplitude of the gravitational wave is modulated, as the detector's sensitivity pattern is not isotropic, so the detected amplitude depends on the location of the source relative to the detector and this location changes due to the rotation of the detector formation about its centre.

Secondly, the detector is moving relative to the source due to the periodic motion of its centre along its orbit around the Sun, Doppler-shifting the frequency of the gravitational wave. This results in a phase modulation of the detector output. The amplitude modulation 
as well as the phase modulation will spread a sharply peaked monochromatic signal into a set of sidebands separated from the carrier at integer multiples of the fundamental frequency $(1 \mathrm{yr})^{-1}$. In the following section a review of the well known matched filtering technique or Wiener optimal filtering will be given, which allows us to extract the wave parameters from the signal, even if stochastic noise has been added. Finally, these techniques are applied to the simulated data for a linearly polarized, monochromatic wave at a frequency of $3 \mathrm{mHz}$ and interpreted to give detailed information about the potential accuracy of the estimation of the angular parameters of the source.

\section{Signal and detector response}

The amplitude modulation can be calculated by transforming the metric tensors

$\mathbf{h}_{\times} \equiv h_{\times}\left(\begin{array}{lll}0 & 1 & 0 \\ 1 & 0 & 0 \\ 0 & 0 & 0\end{array}\right) \quad$ and $\quad \mathbf{h}_{+} \equiv h_{+}\left(\begin{array}{rrr}1 & 0 & 0 \\ 0 & -1 & 0 \\ 0 & 0 & 0\end{array}\right)$

that are defined in the source frame, i.e. a system with the source at its origin and its $z$-axis pointing towards the Sun, into a system that is rigidly fixed to the detector. The transformation is composed of a time-dependent matrix $\mathbf{T}$ and its transpose, each of them being products of simple Euler matrices [1].

The translatory motion of the detector relative to the source leads to a Doppler shift of the frequency of the gravitational wave and therefore to a phase modulation of the signal. If the signal in the inertial frame is purely sinusoidal with frequency $f_{\mathrm{GW}}$, it appears in the detector response as [1]

$$
\begin{aligned}
s_{\mathrm{d}}\left(t_{\mathrm{d}}\right) & =\sin \left(2 \pi f_{\mathrm{GW}} t_{\mathrm{b}}\right) \\
& =\sin (2 \pi f_{\mathrm{GW}} t_{\mathrm{d}}+\underbrace{\frac{2 \pi f_{\mathrm{GW}} R \sin \theta}{c} \cos \left(\frac{2 \pi t}{T}-\phi\right)}_{\Phi(t)}) .
\end{aligned}
$$

Here $t_{\mathrm{b}}$ and $t_{\mathrm{d}}$ are the time-scales with respect to the barycentric system or the system fixed to the detector, respectively. $R$ is the astronomical unit, $\theta$ and $\phi$ are the angular coordinates of the source and $c$ is the speed of light, which is also the propagating speed of the gravitational wave. Equation (2) includes the phase modulation $\Phi(t)$ with a modulation index $m$ of

$$
m=\frac{2 \pi f_{\mathrm{GW}} R \sin \theta}{c} \approx \pi \sin \theta\left(\frac{f_{\mathrm{GW}}}{1 \mathrm{mHz}}\right) .
$$

Combining the above modulations for a gravitational wave which is purely sinusoidal in the barycentric frame causes the strain perceived by the detector to be

$$
\mathbf{H}=\mathbf{T}\left(\begin{array}{cccc}
0 & 0 & 0 & 0 \\
0 & h_{p l} & h_{k r} & 0 \\
0 & -h_{k r} & h_{p l} & 0 \\
0 & 0 & 0 & 0
\end{array}\right) \mathbf{T}^{t} \exp \left\{\mathrm{i}\left[2 \pi f_{\mathrm{GW}} t-\vec{k}_{\mathrm{GW}} \vec{x}+\Phi(t)\right]\right\}
$$

To get the corresponding detector response one has to compute the effect of that gravitational wave on the light travel time in the interferometer arms. Assuming that the metric perturbation is approximately constant during the time any given wavefront 
is present in the apparatus (i.e. $f_{\mathrm{GW}} \tau_{t t} \ll 1$ ) leads to a measurable phase difference in the interferometer arms of [1]

$$
\begin{aligned}
\Delta \Phi & =\frac{2 L \pi}{4 \lambda} \sqrt{3}\left[\left(H_{22}-H_{11}\right) \sqrt{3}+H_{12}+H_{21}\right] \\
& =\frac{L \pi}{\lambda} \sqrt{3}\left[H_{12}-\sqrt{3} H_{11}\right] .
\end{aligned}
$$

In this equation $L$ is the arm length of the interferometer $\left(10^{9} \mathrm{~m}\right)$ and $\lambda$ is the laser wavelength $\left(\approx 10^{-6} \mathrm{~m}\right)$. Due to the motion of the detector a signal that is monochromatic in the source system will now be spread into a set of sidebands in the detector system that contains all the information about the parameters of the gravitational wave. The resulting linear spectrum, divided into real and imaginary parts, is presented in figure 1 . The frequency of the gravitational wave is $3 \mathrm{mHz}$, the polarization is linear (plus).
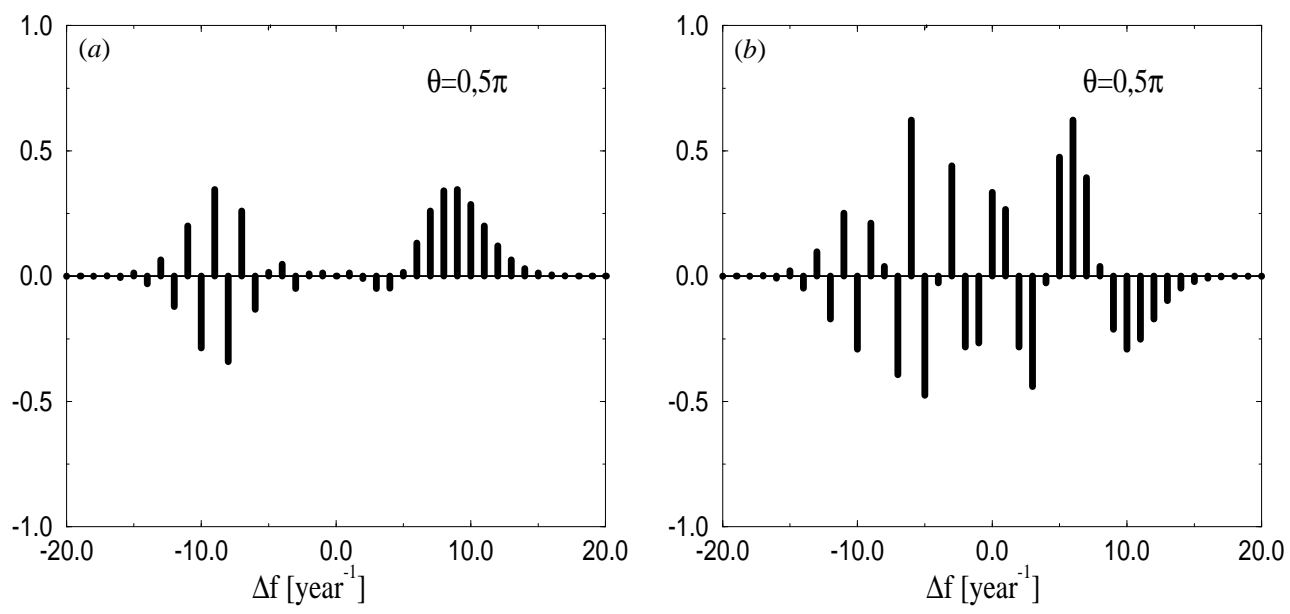

Figure 1. (a) Real part and $(b)$ imaginary part of the detector response to a gravitational wave at $3 \mathrm{mHz}$. The source location is at $(\theta, \phi)=(\pi / 2,0)$.

A gravitational wave signal (cf equation (4)) or the corresponding detector response (cf equation (5)) is characterized by an $M$-dimensional set of parameters $\mu_{1}, \ldots, \mu_{M}$ collectively denoted as $\vec{\mu}_{0}$. Some of these parameters may, for example, be the source location in the sky $\left(\mu_{1}=\theta, \mu_{2}=\phi\right)$ or, for a monochromatic signal, the frequency $\left(\mu_{3}=f_{\mathrm{GW}}\right)$. The problem of parameter estimation is, given a noisy detector response, to determine the value of some or all parameters characterizing the signal and to estimate the measurement error. The noisy detector response $s$ is a time series containing the response (cf equation (5)) plus additional noise $n$, that is assumed to be white, stationary Gaussian noise so it is fully specified by the detectors noise spectrum $\mathcal{S}_{n}$.

The first step in parameter estimation theory is to construct a probability density function (PDF) $\mathcal{P}$. For measured $s\left(\vec{\mu}_{0}\right)$, which is characterized by the real parametrization $\vec{\mu}_{0}, \mathcal{P}$ is a function of a trial set of parameters $\vec{\mu}_{\mathrm{t}}$, in a way that $\mathcal{P}\left(s, \vec{\mu}_{\mathrm{t}}\right) \mathrm{d}^{M} \mu$ is the probability for $\vec{\mu}_{\mathrm{t}}$ to fulfil the condition $\left|\vec{\mu}_{\mathrm{t}}-\vec{\mu}_{0}\right|<|\mathrm{d} \vec{\mu}|$, which means to be inside an $M$-dimensional interval of width $|\mathrm{d} \vec{\mu}|$ centred around $\vec{\mu}_{0}$. If there is no noise contained in $s$, the PDF would of course be a delta function. For noise with the above-mentioned specifications the PDF can be calculated to give [3]

$$
\mathcal{P}\left(s, \vec{\mu}_{\mathrm{t}}\right)=\frac{p\left(\vec{\mu}_{\mathrm{t}}\right)}{\mathcal{N}} \exp \left[2\left\langle s\left(\vec{\mu}_{0}\right), h\left(\vec{\mu}_{\mathrm{t}}\right)\right\rangle-\left\langle h\left(\vec{\mu}_{\mathrm{t}}\right), h\left(\vec{\mu}_{\mathrm{t}}\right)\right\rangle\right] .
$$


Here $\mathcal{N}$ is a normalization constant and $p\left(\vec{\mu}_{\mathrm{t}}\right)$ is an a priori probability for the parametrization $\vec{\mu}_{\mathrm{t}} ; h\left(\vec{\mu}_{\mathrm{t}}\right)$ is a template, which is a calculated detector response for the trial parametrization. The symmetric inner product of $s$ and $h$ is defined as

$$
\langle s, h\rangle=\int_{0}^{\infty} \frac{s(f) h^{*}(f)+h(f) s^{*}(f)}{\mathcal{S}_{n}(f)} \mathrm{d} f .
$$

From this definition it follows that, for a waveform $h\left(\vec{\mu}_{\mathrm{t}}\right)$, the signal-to-noise ratio is given approximately by

$$
\rho\left[\vec{\mu}_{\mathrm{t}}\right] \equiv \frac{\left\langle h\left(\vec{\mu}_{\mathrm{t}}\right), h\left(\vec{\mu}_{\mathrm{t}}\right)\right\rangle}{\operatorname{rms}\left(\left\langle h\left(\vec{\mu}_{\mathrm{t}}\right), n\right\rangle\right)} \approx \sqrt{\left\langle h\left(\vec{\mu}_{\mathrm{t}}\right), h\left(\vec{\mu}_{\mathrm{t}}\right)\right\rangle} .
$$

To evaluate the PDF, that means to measure the values of the parameters, a dataprocessing algorithm has to be chosen. One is the so-called Bayes estimator which will be denoted as $\widehat{\vec{\mu}}[2]$ :

$$
\widehat{\vec{\mu}}=\int \vec{\mu}_{i} \mathcal{P}(s, \vec{\mu}) \mathrm{d}^{M} \mu .
$$

The information about the accuracy of the parameter estimation or the corresponding error of measurement is contained in the variance-covariance matrix

$$
\sigma_{i j}^{2}(\widehat{\vec{\mu}}(s)) \equiv \int\left(\mu_{i}-\widehat{\mu_{i}}\right)\left(\mu_{j}-\widehat{\mu_{j}}\right) \mathcal{P}(s, \vec{\mu}) \mathrm{d}^{M} \mu .
$$

The entries of the vector $\mathrm{d} \vec{\mu}$ that determine the error of measurement and the correlation coefficients $c_{i j}$ are gained from the matrix

$$
\mathrm{d} \mu_{i}=\sqrt{\sigma_{i i}^{2}(\widehat{\vec{\mu}}(s))} \quad c_{i j}=\frac{\sigma_{i j}^{2}(\widehat{\vec{\mu}}(s))}{\sqrt{\sigma_{i i}^{2}(\widehat{\vec{\mu}}(s)) \sigma_{j j}^{2}(\widehat{\vec{\mu}}(s))}} .
$$

\section{Angular resolution}

It is useful to divide the anticipated gravitational waves into three classes [4]: bursts, which last for only a few cycles; stochastic waves which fluctuate stochastically and last for a time which is long compared to an observing run and periodic waves, which are superpositions of sinusoids with frequencies that are more or less constant over times long compared to a typical observing run.

The question of the angular resolution of LISA has to be related to one of these classes. We confined our work to the observation of gravitational waves that are purely monochromatic at $3 \mathrm{mHz}$. We generated a semi-analytic algorithm to calculate the detector response (cf equation (5)) for an arbitrary source location and signal polarization. Using this algorithm we calculated the PDF from (6). In figure 2 the product $\mathcal{P}\left(\int, \vec{\mu}_{\mathrm{t}}\right) \mathrm{d}^{\mathcal{M}} \mu$ is presented for $M=2, \mu_{1}=\theta$ and $\mu_{2}=\phi$. According to (8) the signal-to-noise ratio is $\rho=115$. It turned out that the width of $\mathcal{P}\left(s, \vec{\mu}_{\mathrm{t}}\right) \mathrm{d}^{M} \mu$, or equivalently the errors of measurement corresponding to (11), scales linearly with the inverse of the signal-to-noise ratio.

As can be deduced from figure 2 the errors in $\theta$ and $\phi$ are in the milliradian regime for $\rho=115$. They range from 1 to $8 \mathrm{mrad}$ when the angular parameters are varied. The exact dependence of the errors on source location can be gathered from figure 3. Only values of $\theta$ smaller than $\pi / 2$ an values of $\phi$ smaller than $\pi$ appear in the graphs, because the functions show an appropriate symmetry or periodicity, respectively. 


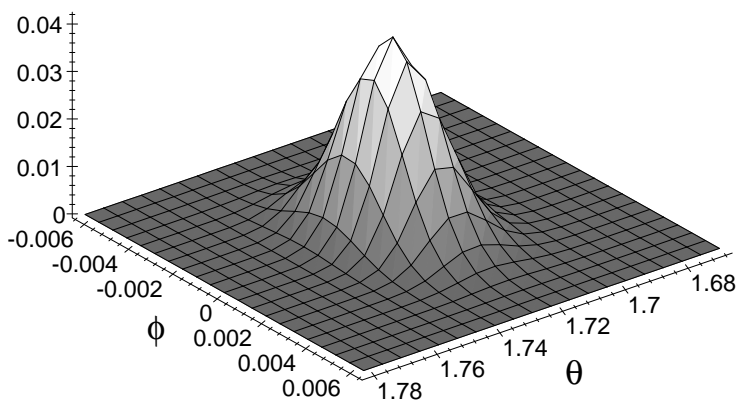

Figure 2. Probability $\mathcal{P}\left(s, \vec{\mu}_{\mathrm{t}}\right) \mathrm{d}^{M} \mu$ for $\vec{\mu}_{0}=(\theta, \phi)=(0.55 \pi, 0)$. The simulation is carried out for a cross-polarized wave at $3 \mathrm{mHz}$ and a signal-to-noise ratio of 115 .
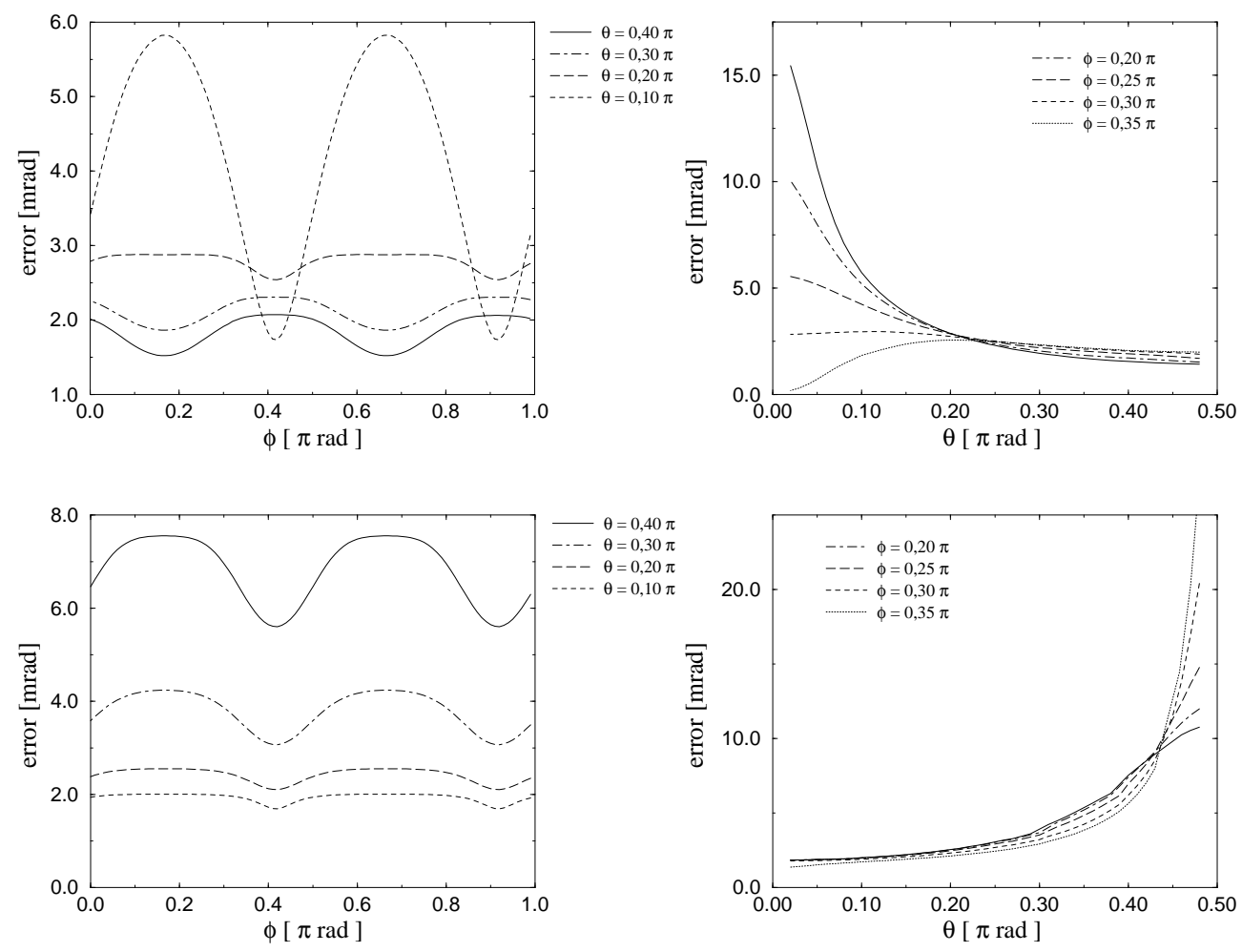

Figure 3. The errors $\mathrm{d} \theta$ (above) and $\mathrm{d} \phi$ (below) corresponding to (11) as functions of source location. The simulation is carried out for a cross-polarized wave at $3 \mathrm{mHz}$ and a signal-to-noise ratio of 115 .

The errors of the parameters $\theta$ and $\phi$ are very weakly correlated for most source locations (cf figure 3). Therefore the canonical way to combine the errors of measurement $\mathrm{d} \theta$ and $\mathrm{d} \phi$ is to present the solid angle of uncertainty

$$
\mathrm{d} \Omega=\sin \theta \mathrm{d} \theta \mathrm{d} \phi .
$$

In the all-sky map (figure 5) we present the total measurement error, when extracting the sources angular parameters from a noisy monochromatic, plus-polarized gravitational wave 


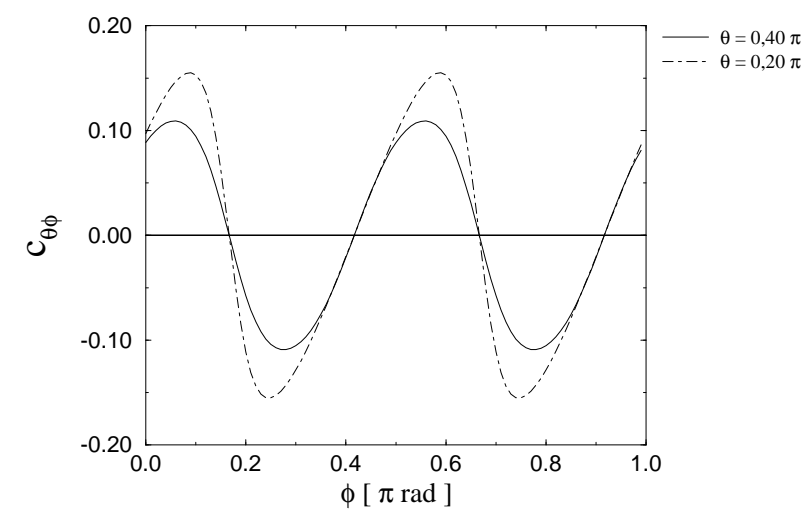

Figure 4. Correlation coefficient of the angular parameters for the detection of a linearly pluspolarized wave at $3 \mathrm{mHz}$.

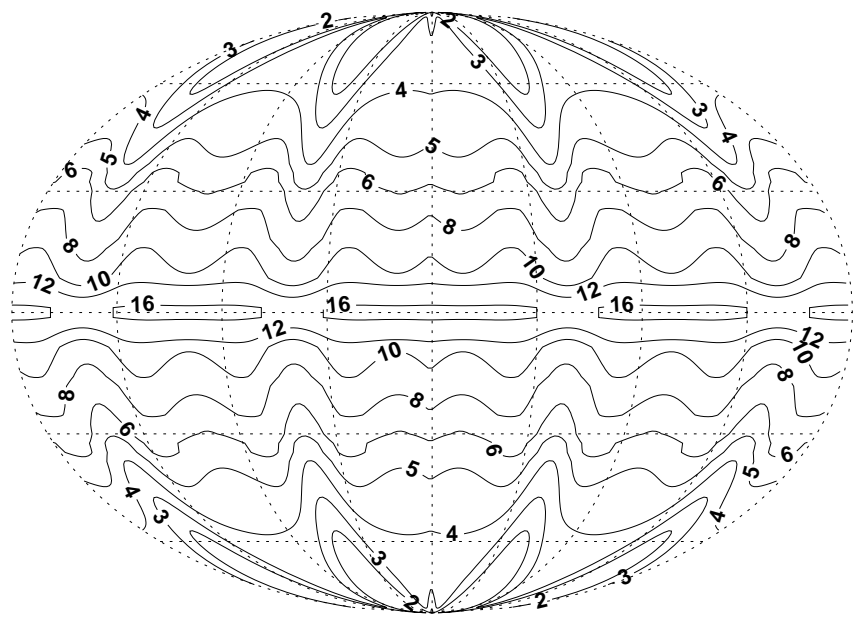

Figure 5. LISA angular resolution all-sky map. The figure shows the solid angle of uncertainty $\mathrm{d} \Omega$ in $\mu \mathrm{sr}$ for the detection of a plus-polarized $3 \mathrm{mHz}$ wave with a signal-to-noise ratio of $\rho=115$.

signal at $3 \mathrm{mHz}$. The signal-to-noise ratio is again $\rho=115$, but now the error scales with the square of the inverse of $\rho$.

\section{References}

[1] Peterseim M, Jennrich O and Danzmann K Class. Quantum Grav. 14 1507-12

[2] Cutler C and Flanagan E E 1994 Phys. Rev. D 49 2658-97

[3] Finn L S 1992 Phys. Rev. D 46 5236-49

[4] Thorne K S 1987300 Years of Gravitation ed S W Hawking and W Israel, pp 330-458

[5] Danzmann and the LISA study team 1996 LISA Pre-phase A report MPQ 209 